\title{
Practicality of Veterans Specific Activity Questionnaire in Evaluation of Exercise Capacity of Community-Dwelling Japanese Elderly
}

\author{
Shinji KOJIMA ${ }^{1}$, Da-Hong WANG ${ }^{1}$, Kimihiko TOKUMORI ${ }^{1}$, Noriko SAKANO ${ }^{1}$, \\ Yukie YAMASAKI ${ }^{1}$, Yoko TAKEMURA ${ }^{1}$, Carmen M. KUROSAWA ${ }^{1}$, Sakiko KANBARA ${ }^{1}$, \\ Takashi OKA ${ }^{2}$, Kohei HARA ${ }^{2}$, Satoru IKEDA ${ }^{3}$ and Keiki OGINO ${ }^{1}$ \\ ${ }^{1}$ Department of Public Health, Graduate School of Medicine, Dentistry and Pharmaceutical Sciences, Okayama University, Okayama, Japan \\ ${ }^{2}$ Yakage Kokuho Hospital, Okayama, Japan \\ ${ }^{3}$ Faculty of Health Sciences, Okayama University Medical School, Okayama, Japan
}

\begin{abstract}
Objective: The aim of this study is to determine whether a questionnaire-based method using the Veterans Specific Activity Questionnaire (VSAQ) is a practical tool for the development of a safe exercise program to prevent a reduction in physical performance.

Methods: One hundred and twenty-one senior residents of Yakage, Okayama, agreed to voluntarily participate in this study. They were asked to complete a questionnaire for information on age, sex, subjective health status, exercise habits and VSAQ. We investigated the relationship between age and exercise capacity predicted by VSAQ (predicted metabolic equivalents (METs)). In addition, for 36 out of the 121 participants, we performed a 6-min walk distance test (6MD) and investigated whether its results correlate with the predicted METs. Furthermore, we prepared a modified VSAQ and examined its practicality in the evaluation of the exercise capacity of Japanese elderly $(n=50)$.

Results: We found that the predicted METs correlate well with age. Habitual exercise and subjective health status did not affect the predicted METs. A significant correlation was observed between the predicted METs and the results of $6 \mathrm{MD}(\mathrm{r}=0.56, \mathrm{p}<0.001)$. We also found that certain activities included in the original VSAQ are unfamiliar to Japanese elderly; thus, we made a few modifications to the original VSAQ in order to evaluate the physical fitness of Japanese elderly. The number of inadequate answers was reduced by employing the modified VSAQ.

Conclusion: These findings imply that the modified VSAQ is useful in evaluating the exercise capacity of Japanese elderly adequately and is a practical scale for safe exercise.
\end{abstract}

Key words: VSAQ, exercise capacity, noninvasive evaluation, Japanese elderly, community health service

\section{Introduction}

In Japan, the population of older adults is growing rapidly. Recently, the activities on fall prevention and strength training, as approaches to preventing the reduction in physical performance, have been widely performed nationwide (1), and most of these activities are strength training-based exercise interventions that employ certain elements of aerobic exercise and

Received May 17, 2006/Accepted Sep. 7, 2006

Reprint requests to: Shinji KOJIMA

Department of Public Health, Graduate School of Medicine, Dentistry and Pharmaceutical Sciences, Okayama University, 2-5-1 Shikata-cho, Okayama 700-8558, Japan

TEL: +81(86)235-7184, FAX: +81(86)226-0715

E-mail: skojima@md.okayama-u.ac.jp flexibility training (2). Many studies have found that having knowledge of fall prevention and adopting exercise with various elements are practical in preventing falls $(3,4)$. Therefore, improvements in the various types of exercise capacity as well as muscle force are necessary for preventing a reduction in physical performance. However, safety is a big concern in the implementation of physical training among the elderly, and an individualized intervention program based on the evaluation results of the individual exercise capacity of the elderly is desirable.

Maximum oxygen uptake is an indicator of exercise capacity. However, an exercise test has to be carried out for the measurement of maximum oxygen uptake. Since the elderly potentially have cardiopulmonary disorders, much attention should be paid to performing exercise test safely (5); for this reason, a considerable amount of labor is required while evalu- 
ating of exercise capacity in a community health service program for many elderly at the same time and at a place far from medical facilities.

Thus far, several methods have been used to evaluate individual exercise capacity without performing the exercise test, for example, the Borg scale (rating of perceived exertion) and methods that enable the prediction of metabolic equivalents (METs) from age $(6,7)$. In addition, questionnaire-based evaluations methods, such as those using the Veterans Specific Activity Questionnaire (VSAQ) and The Duke Activity Status Index (DASI), have been widely used in many countries and introduced into the textbook of the American College of Sports Medicine (8-10). In Japan, these questionnaire-based methods have been introduced into a number of exercise guidebooks for the assessment of cardiopulmonary function in circulatory disease and the evaluation of functional capacity recovery in the rehabilitation field, but whether these methods are applicable to Japanese elderly over the age of 75 is unclear $(11,12)$.

The aim of this study is to examine whether a questionnaire-based method using VSAQ is a practical tool for the development of a safe exercise program to prevent reduction in the physical performance of the elderly in Yakage town, where $30.1 \%$ of the residents were aged 65 and over in August 2005.

\section{Subjects and Methods}

\section{Subjects}

The study population consisted of 121 senior citizens who attended a health promotion class at the Yakage Town Health Management Center in Okayama prefecture of Japan from August 2004 to July 2005 and who agreed to voluntarily participate in this study. All participants were informed of the purpose of the study and signed an informed consent form. This study obtained the approval of the Ethical Committee for Epidemiologic Studies of Okayama University Graduate School of Medicine, Dentistry and Pharmaceutical Sciences.

\section{VSAQ}

VSAQ, developed by Myers et al., is a very simple and easy questionnaire-based method that enables the evaluation of the exercise capacity of middle-aged and elderly people, and it has been used widely in the USA.

The use of VSAQ allows the determination of different intensities of daily activities with corresponding MET in an increasing order. The scale ranges from 1 MET to 13 METs (Appendix 1). The participants were asked to choose the MET that reflected the activity with the highest intensity that the participants are able to do routinely, and this MET was expressed as "VSAQ maximum". Then, we substituted this value for the following regression equation developed by Myers et al. (9) to predict the exercise capacity of the participants.

Predicted METs $=4.74+0.97 \times($ VSAQ maximum $)-0.06 \times($ age $)$

In this study, we used the self-administered Japanese version of VSAQ. We explained the meaning of each question to the participants orally and let them reply to it after understanding the questionnaire sufficiently. The participants were also asked to identify the activities in a list categorized in term of their MET (1-13) (Appendix 1) into that they are able to do routinely or they are unable to do.

\section{6-min walk distance test (6MD)}

Thirty-six females of the 121 elderly agreed to participate in a $6 \mathrm{MD}$ for the determination of their exercise capacity, in particular their aerobic exercise capacity. We then investigated the correlation between $6 \mathrm{MD}$ and VSAQ results. The results of $6 \mathrm{MD}$, which is a test developed by Butland et al. by improving the 12-min walk distance test, highly correlate with maximum oxygen uptake (13-16). Such a test indicates the distance (m) that a person can walk in 6 min when walking quickly on a flat surface. Solway et al. suggested that $6 \mathrm{MD}$ is a superior method in terms of handiness, low burden to a subject and easiness in evaluating activities of daily life (17). We carried out 6MD according to the guidelines of the American Thoracic Society (18). 6MD was performed indoors, along a flat and enclosed corridor with a hard surface. The walking course was $30 \mathrm{~m}$ in length and was marked every $3 \mathrm{~m}$ with orange traffic cones.

\section{Questionnaire survey}

The participants were asked to complete a self-administered questionnaire for information on age, sex, subjective health status, exercise and physical activities.

Information on subjective health status was obtained from the results of the participants choosing one of the following five choices: "Very healthy", "Kind of healthy", "I'm not sure", "Kind of unwell", and "Unwell". Information on exercise and physical activities was obtained from the results of the participants choosing one of the following four choices: "Do exercise regularly", "Do exercise occasionally", "Did exercise before", and "Did not do exercise at all". Regular exercise was defined as a 30-min of exercise performed 2 days or more per week for more than one year.

\section{Preparation of Japanese elderly-targeted VSAQ (modified VSAQ)}

In the VSAQ developed by Myers et al. (original VSAQ), certain daily activities were unfamiliar to Japanese elderly. Therefore, we made a few modifications to the original VSAQ in order to evaluate the physical fitness of Japanese elderly precisely (Appendix 2). In the original VSAQ, 13 MET items were enumerated for each MET. In the modified VSAQ, two doctors, two physical therapists, and one public health nurse reviewed the questions and then changed certain activity items to those familiar to Japanese elderly. These newly added activities were in agreement with each MET and were chosen on the basis of the Compendium of Physical Activities by Ainsworth et al. (19). We examined the practicality of the modified VSAQ in 50 females who agreed to participate in this study and also carried out 6MD on them to examine the relationship between the results of the modified VSAQ and those of 6MD.

\section{Calculation of defection rate}

We also determined the difference in answer state between the original VSAQ and the modified VSAQ using the defection rate of each MET using the following formula: Defection rate $=$ $[(\mathrm{A}-\mathrm{B}) / \mathrm{A}] \times 100 \%$, where $\mathrm{A}$ is the number of participants 
who answered that the intensity of their VSAQ maximum was higher than that of each corresponding MET item, B is the number of participants who answered that they are able to do the activities for each corresponding MET.

\section{Statistical analysis}

The Mann-Whitney $U$ test was used to determine the difference between the groups, and the relationships between factors were analyzed using the Spearman correlation coefficient with a $95 \%$ confidence interval $(95 \% \mathrm{CI})$. Calculations were performed with SPSS ver. 12.0J and Microsoft Excel. Values of $\mathrm{p}<0.05$ were considered statistically significant.

\section{Results}

\section{Description of study participants}

Table 1 shows characteristics of the participants. In regard to subjective health status, most of the participants $(71.1 \%)$ answered "Very healthy" or "Kind of healthy", and among them, $44.6 \%$ exercised for more than 30 minutes 2 days or more per week for more than one year.

\section{Distribution of predicted METs determined by VSAQ}

Distributions of the predicted METs are shown in Fig. 1. The mean of the predicted METs for the 121 participants was 7.61 METs (range 2.85-12.9). The predicted METs of 14 people $(11.6 \%)$ were beyond $10 \mathrm{METs}$, which is too high for the elderly in terms of exercise capacity.

\section{Relationship between predicted METs and other factors}

3-1) Relationship between predicted METs and age

Concerning the 121 participants, the correlation between the predicted MET and age was statistically significant $(\mathrm{r}=-0.33$, $\mathrm{p}<0.01,95 \% \mathrm{CI} ; 0.16-0.48)$. The predicted METs of 38 males correlated with age $(\mathrm{r}=-0.44, \mathrm{p}<0.01,95 \% \mathrm{CI} ; 0.14-0.67)$, and those of 83 females also correlated with age $(\mathrm{r}=-0.33, \mathrm{p}<0.01$, $95 \% \mathrm{CI} ; 0.12-0.51$ ).

3-2) Relationship among sex, subjective health status, exercise and physical activities, and VSAQ results

Comparisons of the predicted METs by sex, subjective health status and exercise habits are shown in Table 2. In regard

Table 1 Characteristics of participants

\begin{tabular}{lccc}
\hline & $\begin{array}{c}\text { Total } \\
(\mathrm{n}=121)\end{array}$ & $\begin{array}{c}\text { Elderly under age of } 75 \\
(\mathrm{n}=52)\end{array}$ & $\begin{array}{c}\text { Elderly over age of } 75 \\
(\mathrm{n}=69)\end{array}$ \\
\hline Sex & & & \\
Male & $38(31.5 \%)$ & $15(28.8 \%)$ & $23(33.3 \%)$ \\
Female & $83(68.5 \%)$ & $37(71.2 \%)$ & $46(66.7 \%)$ \\
Age (year) & $75.3 \pm 6.22$ & $69.7 \pm 2.61$ & $79.5 \pm 4.61$ \\
& $(65 \sim 95)^{*}$ & $(65 \sim 74)^{*}$ & $(75 \sim 95)^{*}$ \\
Exercise habit & & & \\
"Do exercise regularly" & $54(44.6 \%)$ & $24(46.2 \%)$ & $30(43.5 \%)$ \\
"Do exercise occasionally" & $45(37.2 \%)$ & $16(30.8 \%)$ & $29(42.0 \%)$ \\
"Did exercise before" & $13(10.7 \%)$ & $7(13.5 \%)$ & $6(8.7 \%)$ \\
"Did not do exercise at all" & $9(7.4 \%)$ & $5(9.6 \%)$ & $4(5.8 \%)$ \\
Subjective health status & & & $14(20.3 \%)$ \\
"Very healthy" & $18(14.9 \%)$ & $4(7.7 \%)$ & $31(44.9 \%)$ \\
"Kind of healthy" & $68(56.2 \%)$ & $4(71.2 \%)$ & $8(11.6 \%)$ \\
"I'm not sure" & $12(9.9 \%)$ & $6(11.5 \%)$ & $10(14.5 \%)$ \\
"Kind of unwell" & $16(13.2 \%)$ & $1(1.9 \%)$ & $4(5.8 \%)$ \\
"Unwell" & $5(4.1 \%)$ & 0 & $(0 \%)$ \\
No answer & $2(1.7 \%)$ & & $2.9 \%)$ \\
\hline
\end{tabular}

* Mean \pm standard deviation (range).
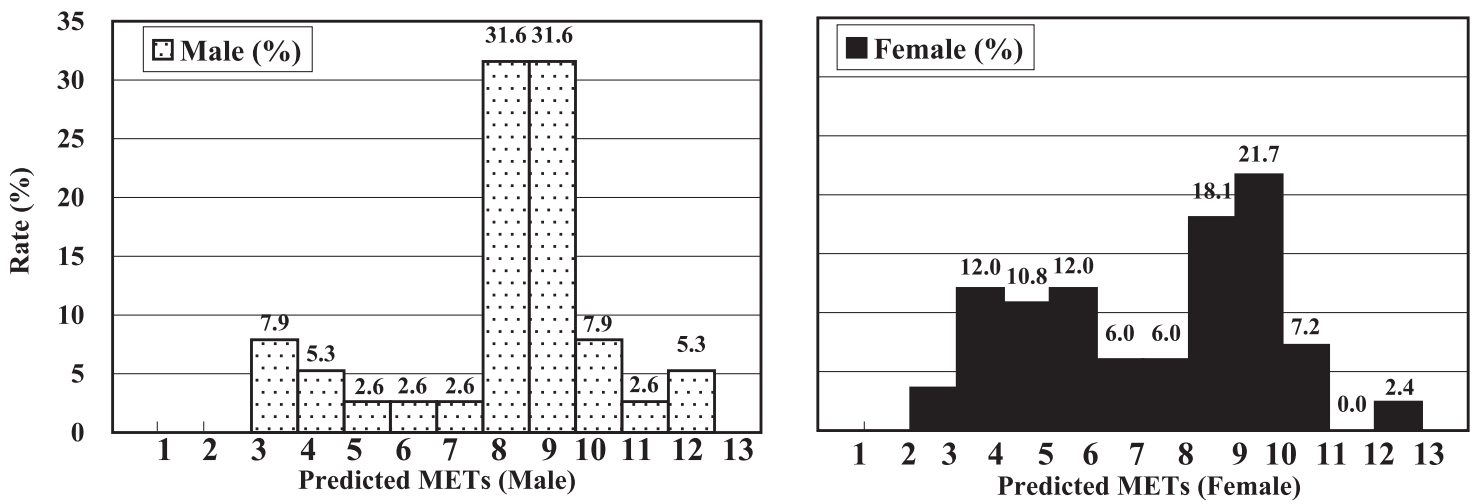

Fig. 1 Distribution of predicted METs based on VSAQ results. 
to subjective health status, persons who chose "Very healthy" and "Kind of healthy" were classified into the "Feeling healthy" group. People who chose "I'm not sure", "Kind of unwell" and "Unwell" were classified into the "Not feeling healthy" group. There was no statistically significant difference in the mean of the predicted METs between the groups in regard to sex, subjective health status and exercise habits, but exercise capacity was highly predicted in the "Not feeling healthy" male group. However, as for all the participants $(n=121)$, there was no difference in predicted MET in regard to subjective health status $(p=0.399)$.

3-3) Relationship between predicted METs and 6MD results $6 \mathrm{MD}$ was carried out on 36 females who agreed to take the test. The mean age of the participants was 72.9 (range 65-91).
There was a significant correlation between the predicted METs and $6 \mathrm{MD}$ results $(\mathrm{r}=0.56, \mathrm{p}<0.001,95 \% \mathrm{CI} ; 0.28-0.75)$. The predicted METs highly correlated with $6 \mathrm{MD}$ results in regard to age $(\mathrm{r}=-0.36, \mathrm{p}<0.05,95 \% \mathrm{CI} ; 0.04-0.62)$.

\section{Practicality of the original VSAQ in Japanese elderly}

VSAQ consists of different intensities of daily activities corresponding to 1 MET-13 METs values in an increasing order. Therefore, we note that the participants are able to do all the activities with intensities lower than VSAQ maximum. However, 66 participants out of the 121 (54.5\%) answered that they are unable to do some activities with intensities lower than VSAQ maximum. Fig. 2 shows the responses of the participants to each VSAQ MET. Black parts indicate that the participants were able to do the activities with intensities corresponding to

Table 2 Comparison of predicted METs with regard to sex, subjective feeling of health and exercise habit

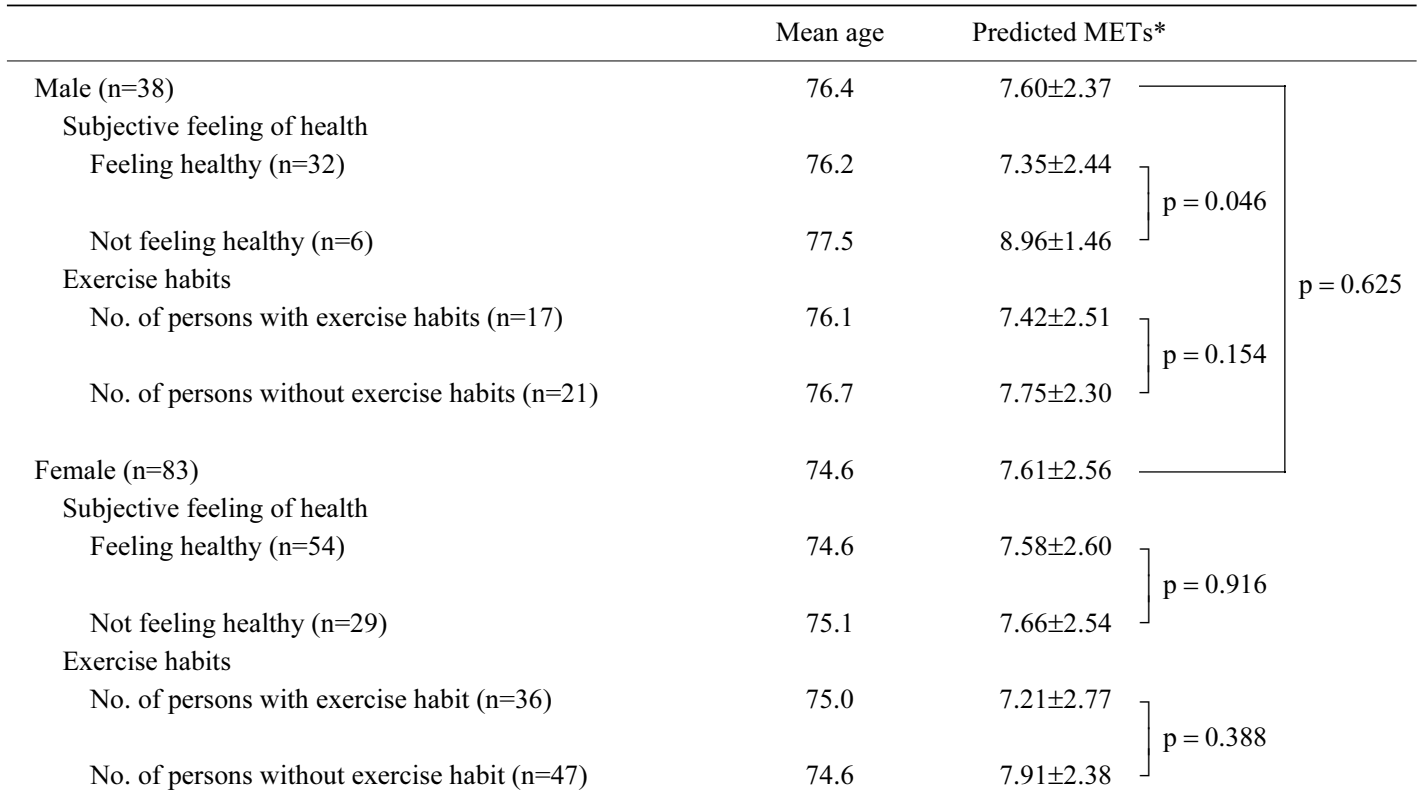

* Data are expressed as mean \pm standard deviation. The Mann-Whitney $U$ test was used for comparison. Persons who chose "Very healthy" and "Kind of healthy" were classified into the "Feeling healthy" group in regard to subjective health status.

Exercise habits were defined as exercising more than $30 \mathrm{~min}$ of two days or more per week for more than one year.

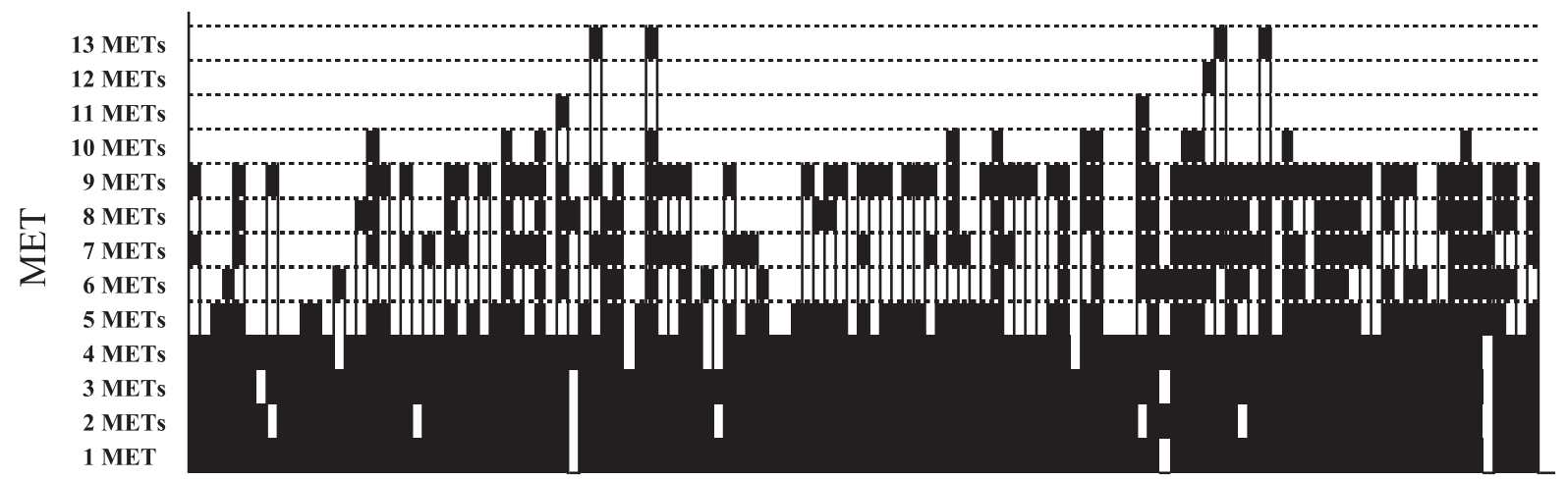

Case $(\mathrm{n}=121)$

Fig. 2 Response to each MET item of original VSAQ by each participant. The vertical axis denotes a MET item, and the horizontal axis denotes a case. Black parts indicate the participants answering that it was possible for them to do the activities with intensities corresponding to a certain MET, whereas blank parts indicate the participants who thought it was impossible for them to do the activities. The items corresponding to 5 METs- 8 METs and 10 METs-12 METs were notable. 


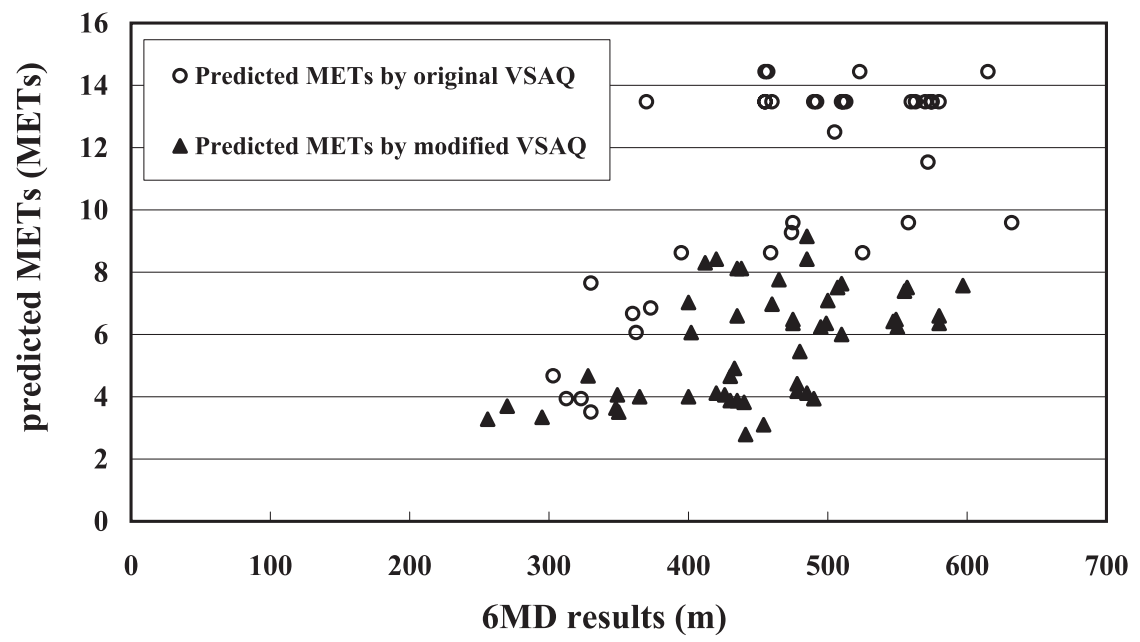

Fig. 3 Scatter diagram of predicted METs by VSAQ and 6MD. The METs predicted by the original VSAQ $(r=0.56, p<0.001,95 \%$ CI; $0.28-$ $0.75)$ and those by the modified VSAQ ( $r=0.48, \mathrm{p}<0.001,95 \% \mathrm{CI} ; 0.23-0.67)$ correlated relatively well with $6 \mathrm{MD}$ results.

a certain MET. The black part on the top indicates VSAQ maximum. On the other hand, blank parts indicate that the participants were unable to do the activities. The activities corresponding to 5 METs- 8 METs and 10 METs-12 METs were in the blank parts. However, the activities corresponding to 9 METs were mostly in the black parts.

\section{Evaluation of Japanese elderly-targeted VSAQ}

The above-mentioned results demonstrated that the original VSAQ inadequate in evaluating the physical fitness of Japanese elderly, because it was developed on the basis of Western-style activities. Therefore, we developed a Japanese elderly-targeted VSAQ (modified VSAQ) in which we replaced questions with the questions familiar to Japanese elderly (Appendix 2).

Fifty females (mean age, 74.0) were subjected to the modified VSAQ and 6MD. We found a significant correlation between predicted METs by the modified VSAQ and 6MD results. The relationship between $6 \mathrm{MD}$ results and predicted METs by the original VSAQ, and between 6MD results and predicted METs by the modified VSAQ are shown in Fig. 3. The prediction of exercise capacity by the original VSAQ was not accurate for certain cases that were over 10 METs, but

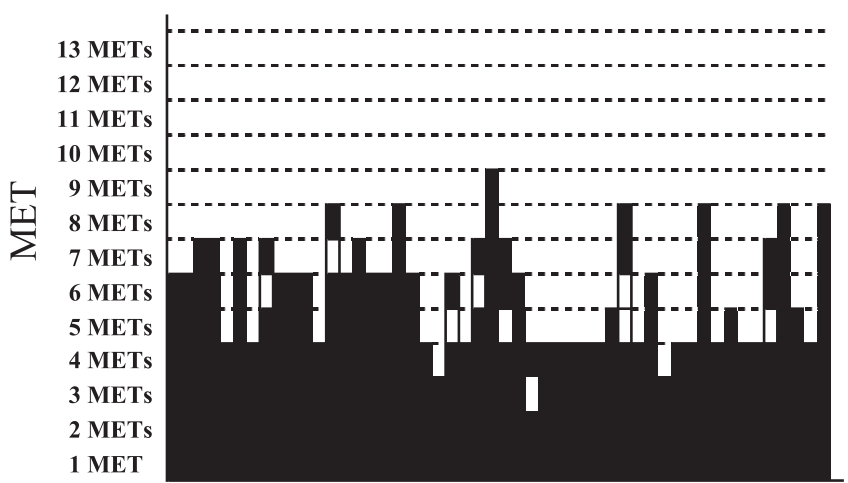

Case $(n=50)$

Fig. 4 Response to each MET of modified VSAQ by each participant.

this was not found in the case of using the modified VSAQ. Furthermore, the response defection to the questions of the modified VSAQ was observed in only 8 participants out of the $50(16.0 \%)$ (Fig. 4), which was low in comparison with that of the original VSAQ $(54.5 \%)$. Table 3 shows the differences in

Table 3 Differences in answer state and defection rate of each MET between two questionnaires

\begin{tabular}{|c|c|c|c|c|c|c|c|c|c|c|c|c|c|}
\hline & \multicolumn{13}{|c|}{ MET item } \\
\hline & 1 & 2 & 3 & 4 & 5 & 6 & 7 & 8 & 9 & 10 & 11 & 12 & 13 \\
\hline \multicolumn{14}{|l|}{ Original VSAQ $(n=121)$} \\
\hline Numbers supposed to be answered (A) & 121 & 121 & 121 & 119 & 99 & 86 & 80 & 75 & 71 & 18 & 7 & 5 & 4 \\
\hline Numbers actually answered (B) & 117 & 113 & 116 & 115 & 68 & 38 & 53 & 41 & 71 & 13 & 2 & 1 & 4 \\
\hline Difference $(A-B)$ & 4 & 8 & 5 & 3 & 30 & 47 & 26 & 33 & 0 & 5 & 5 & 4 & 0 \\
\hline Defection rate $[(\mathrm{A}-\mathrm{B}) / \mathrm{A}] \times 100 \%$ & 3.3 & 6.6 & 4.1 & 2.5 & 30.3 & 54.7 & 32.5 & 44.0 & 0.0 & 27.8 & 71.4 & 80.0 & - \\
\hline \multicolumn{14}{|l|}{ Modified VSAQ $(n=50)$} \\
\hline Numbers supposed to be answered (A) & 50 & 50 & 50 & 48 & 30 & 28 & 15 & 7 & 1 & 0 & 0 & 0 & 0 \\
\hline Numbers actually answered (B) & 50 & 50 & 49 & 48 & 26 & 25 & 14 & 7 & 1 & 0 & 0 & 0 & 0 \\
\hline Difference $(A-B)$ & 0 & 0 & 1 & 0 & 4 & 3 & 1 & 0 & 0 & 0 & 0 & 0 & 0 \\
\hline Defection rate $[(\mathrm{A}-\mathrm{B}) / \mathrm{A}] \times 100 \%$ & 0.0 & 0.0 & 2.0 & 0.0 & 13.3 & 10.7 & 6.7 & 0.0 & 0.0 & 0.0 & 0.0 & 0.0 & - \\
\hline
\end{tabular}

A, the number of participants who answered that the intensity of their VSAQ maximum was higher than that of each corresponding MET item.

$\mathrm{B}$, the number of participants who answered that they are able to do the activities for each corresponding MET. 
responses to each question between the original VSAQ and the modified VSAQ. For the modified VSAQ, the defection rate of each corresponding MET decreased greatly.

\section{Discussion}

We found that VSAQ has the potential to be used in evaluating the exercise capacity of the elderly when certain questions are placed with those familiar to Japanese elderly.

In regard to the validity of VSAQ in the evaluation of exercise capacity, we examined how the METs predicted by VSAQ correlated with 6MD results, because 6MD is one of the scales for measuring the aerobic exercise capacities of various age groups (20). Since the METs predicted by VSAQ in this study correlated well with the 6MD results in the case of females, VSAQ can be used as a method of evaluating the exercise capacity of the elderly. With respect to gender difference, men generally surpass women in exercise capacity (21). However, we found that there is no difference in predicted MET between men and women in the same age category. This result suggests that the exercise capacity of women is estimated to be higher than the actual exercise capacity by VSAQ. Moreover, we found that habitual exercise and subjective health status do not affect the predicted METs, but exercise capacity was highly predicted in the "Not feeling healthy" male group. However, there was no difference in predicted MET in regard to subjective health status among any of the participants. Since there were only 6 participants belonging to the "Not feeling healthy" male group, the present results should be considered with caution, and further study is required to confirm these results. We predicted that people with confidence in their health would highly rate their exercise capacity. However, we did not find a significant contribution of the subjective factors to the predicted METs. Furthermore, as regards the correlation with 6MD results, the predicted METs $(\mathrm{r}=0.56)$ had a higher correlation than age $(\mathrm{r}=-0.36)$, indicating that the METs predicted by VSAQ are more practical in predicting exercise capacity than age alone. However, the results showed that the mean age of these participants is 75.3 years old and that the mean predicted MET is 7.61 METs, implying that exercise capacity is predicted highly by this evaluation method. A possible explanation for the above-mentioned result is that most of the participants who had a highly subjective health status and exercised regularly participated in this study. That is, the predicted METs may tend to be highly evaluated in a very motivated group of participants. Therefore, when we prescribe an exercise program for the general population of elderly using VSAQ, we should set the activity intensity lower than the target intensity.

In this study, we found that certain activities in the original VSAQ are unfamiliar to Japanese elderly, because they were based on Western-style activities. Fig. 2 demonstrates that the activities corresponding to 5 METs- 8 METs and 10 METs- 12 METs chosen by each participant are mostly in the blank parts: nevertheless, these activity items had intensities lower than that of the VSAQ maximum of the participants. The activities corresponding to 5 METs- 8 METs, such as dancing, car washing and pushing power mower are common in Westernstyle lives; therefore, it is important to examine their suitability to Japanese elderly. Furthermore, concerning the activities corresponding to 10 METs-12 METs, three people out of four who answered that they were unable to do activities of 10 METs, 11 METs and 12 METs chose the items of 13 METs, implying that Japanese elderly can perform activities of 13 METs. A similar case was also found in the activities of 9 METs. On the basis of these findings, we recognized the necessity of modifying the activities with intensities of 5 METs and higher in the original VSAQ in order to accurately evaluate the exercise capacity of Japanese elderly.

In the modified VSAQ, we changed certain activities to those familiar to Japanese elderly the basis of the Compendium of Physical Activities (19). For example, we changed "social dancing" to "playing with animals" in 5 METs, and we replaced "mow lawn with push mower" with "bicycling" in 6 METs. In addition, "basketball", "cross country ski", "backpacking” were changed to "running", "swimming". In the case of females, the number of defection items was reduced by such modifications, the mean exercise capacity overpredicted by the original VSAQ was reduced to 5.69 METs (range 2.79-9.15) by the modified VSAQ, and the cases corresponding to 10 METs-13 METs that were unrealistically highly predicted were eliminated. These findings imply that the modified VSAQ is adequate in evaluating the exercise capacity of Japanese elderly who reside in the rural district and keep high daily life activity levels, and is a practical scale for safe exercise.

The present findings have several limitations. First, the sample size was small and the data were collected from a highly motivated group of participants. These facts limit the ability to generalize the present findings. Second, we did not directly measure the maximum oxygen uptake because it entails physical demands on elderly participants; instead, we employed $6 \mathrm{MD}$, a test that highly correlates with the maximum oxygen uptake, to measure the exercise capacity of the participants. Third, the participants who performed the modified VSAQ did not completely correspond with the participants who performed the original VSAQ. Therefore, we cannot evaluate the two questionnaires precisely only using the number of defection items. Further testing with the general population of elderly is required to understand the broader applicability of the modified VSAQ.

\section{Acknowledgments}

We would like to express our thanks to the staff of the Yakage Town Health Management Center who cooperated in this study. We are extremely grateful to the late Professor Shohei Kira for his valuable instruction. 


\section{References}

( 1 ) Suzuki T, Kim H, Yoshida H, Ishizaki T. Randomized controlled trial of exercise intervention for the prevention of falls in community-dwelling elderly Japanese women. J Bone Miner Metab. 2004;22:602-611.

( 2 ) Province MA, Hadley EC, Hornbrook MC, Lipsitz LA, Miller JP, Mulrow CD, et al. The effects of exercise on falls in elderly patients. A preplanned meta-analysis of the FICSIT Trials. JAMA. 1995;273:1341-1347.

( 3 ) Gillespie LD, Gillespie WJ, Robertson MC, Lamb SE, Cumming RG, Rowe BH. Interventions for preventing falls in elderly people (Cochrane Review). Cochrane Library. 2001;4.

( 4 ) Chang JT, Morton SC, Rubenstein LZ, Mojica WA, Maglione $\mathrm{M}$, Suttorp MJ, et al. Interventions for the prevention of falls in older adults: systematic review and meta-analysis of randomised clinical trials. BMJ. 2004;328:680.

( 5 ) Kubo M, Kiyohara Y, Kato I, Tanizaki Y, Arima H, Tanaka $\mathrm{K}$, et al. Trends in the incidence, mortality, and survival rate of cardiovascular disease in a Japanese community: the Hisayama study. Stroke. 2003;34:2349-2354.

( 6 ) Borg G. Perceived exertion as an indicator of somatic stress. Scand J Rehabil Med. 1970;23:92-93.

( 7 ) Dehn MM, Bruce RA. Longitudinal variations in maximal oxygen intake with age and activity. J Appl Physiol. 1972; 33:805-807.

( 8 ) Hlatky MA, Boineau RE, Higginbotham MB, Lee KL, Mark DB, Califf RM, et al. A brief self-administered questionnaire to determine functional capacity (The Duke Activity Status Index). Am J Cardiol. 1989;15:651-654.

(9) Myers J, Do D, Herbert W, Ribisl P, Froelicher VF. A nomogram to predict exercise capacity from a specific activity questionnaire and clinical data. Am J Cardiol. 1994;73:591-596.

(10) Barry AF, Mitchell HW, Edward TH. ACSM's Guidelines for Exercise Testing and Prescription, 6th ed. American College of Sports Medicine. Philadelphia, Pennsylvania: Lippincott Williams \& Wilkins; 2000.

(11) Pierson LM, Norton HJ, Herbert WG, Pierson ME, Ramp
WK, Kiebzak GM, et al. Recovery of self-reported functional capacity after coronary artery bypass surgery. Chest. 2003; 123:1367-1374.

(12) Cook JW, Pierson LM, Herbert WG, Norton HJ, Fedor JM, Kiebzak GM, et al. The influence of patient strength, aerobic capacity and body composition upon outcomes after coronary artery bypass grafting. Thorac Cardiovasc Surg. 2001;49:8993.

(13) Cooper KH. A means of assessing maximal oxygen intake: correlation between field and treadmill testing. JAMA. 1968;203:201-204.

(14) Enright PL, McBurnie MA, Bittner V, Tracy RP, McNamara $\mathrm{R}$, Arnold A, et al. The 6-min walk test: a quick measure of functional status in elderly adults. Chest. 2003;123:325-327.

(15) Cahalin L, Papagianopoulos P, Prevost S, Wain J, Ginns L. The relationship of 6-min walk test to maximal oxygen consumption in transplant patients with end-stage lung disease. Chest. 1995;108:452-459.

(16) Butland RJ, Pang J, Gross ER, Woodcock AA, Geddes DM. Two-, six-, and 12-minute walking tests in respiratory disease. BMJ. 1982;284:1607-1609.

(17) Solway S, Brooks D, Lacasse Y, Thomas S. A qualitative systematic overview of the measurement properties of functional walk test used in the cardiorespiratory domain. Chest. 2001;119:256-270.

(18) ATS statement. Guidelines for the six-minute walk test. Am J Respir Crit Care Med. 2002;166:111-117.

(19) Ainsworth BE, Haskell WL, Whitt MC, Irwin ML, Swartz AM, Strath SJ, et al. Compendium of physical activities: an update of activity codes and MET intensities. Med Sci Sports Exerc. 2000;32:498-504.

(20) Enright PL, Sherrill DL. Reference equations for the sixminute walk in healthy adults. Am J Respir Crit Care Med. 1998;158:1384-1387.

(21) Astrand PO, Ryhming I. A nomogram for calculation of aerobic capacity (physical fitness) from pulse rate during submaximal work. J Appl Physiol. 1954;7:218-221. 


\section{Appendix 1}

Original VSAQ*. Draw one line below the activities you are able to do routinely with minimal or no symptoms, such as shortness of breath, chest discomfort and fatigue.

\begin{tabular}{cl}
\hline & Activities \\
\hline 1 MET & Eating, getting dressed, working at a desk \\
2 METs & Taking a shower, walking down eight steps \\
3 METs & Walking slowly on a flat surface for one or two blocks $(150-200 \mathrm{~m})$, a moderate amount of work around the house, like vacuuming, \\
& sweeping the floors or carrying groceries \\
4 METs & Light yard work, i.e., raking leaves, weeding or pushing a power mower, painting or light carpentry \\
5 METs & Walking briskly, i.e., four miles in one hour $(6.4 \mathrm{~km} / \mathrm{hour})$, social dancing, washing the car \\
$6 \mathrm{METs}$ & Play nine holes of golf carrying your own club, heavy carpentry, mow lawn with push mower \\
7 METs & Perform heavy outdoor work, i.e., digging, spading soil, play tennis (singles), carry 60 pounds $(27 \mathrm{~kg})$ \\
$8 \mathrm{METs}$ & Move heavy furniture, jog slowly climb stairs quickly, carrying 20 pounds $(9 \mathrm{~kg})$ upstairs \\
9 METs & Bicycling at a moderate pace, sawing wood, jumping rope (slowly) \\
10 METs & Brisk swimming, bicycle up a hill, walking briskly uphill, jog six miles per hour $(9.6 \mathrm{~km} / \mathrm{h})$ \\
11 METs & Cross country ski, play basketball (full court) \\
$12 \mathrm{METs}$ & Running briskly, level ground, eight minutes per mile $(12.8 \mathrm{~km} / \mathrm{h})$, continuously \\
13 METs & Any competitive activity, including those which involve intermittent sprinting, running competitively, rowing, backpacking \\
\hline
\end{tabular}

* From Myers et al. (9).

\section{Appendix 2}

Modified VSAQ. Draw one line below the activities you are able to do routinely with minimal or no symptoms, such as shortness of breath, chest discomfort and fatigue.

\begin{tabular}{cl}
\hline & Activities* \\
\hline 1 MET & Eating, getting dressed, working at a desk \\
2 METs & Taking a shower, walking down eight steps \\
3 METs & Walking slowly on a flat surface for one or two blocks $(150-200 \mathrm{~m})$, performing a moderate amount of work around the house, such as \\
& vacuuming, sweeping the floors or carrying groceries \\
4 METs & Performing light yard work, i.e., raking leaves, weeding or pushing a power mower, painting or light carpentry \\
5 METs & Playing with animals, vigorously walking, walking downstairs or standing, carrying objects about 25-49 lbs (10-20 kg), walking \\
& 4.0 mph (6.4 km/h) on a level, hard surface, briskly. \\
6 METs & Bicycling 10-11.9 mph $(16$ km/h), shoveling snow by hand, hiking, cross country \\
7 METs & Forestry, sawing by hand, jogging in general \\
8 METs & Jumping rope slowly, carrying a 25-49-lbs (10-20-kg) load upstairs, rock or mountain climbing \\
9 METs & Moving household items upstairs, carrying boxes or furniture, climbing hills with 42-lb load \\
10 METs & Running 6 mph (10 km/h), or practicing judo, jujitsu, karate, kick boxing, or tae kwan do \\
11 METs & Rock climbing, ascending rock, swimming butterfly, in general \\
12 METs & Jumping rope, fast, carrying 74-Ibs (34-kg) load upstairs \\
13 METs & Skin diving at a moderate pace, running 7.8 mph $(12.5$ km/h) \\
\hline
\end{tabular}

* The activities corresponding to 5 METs-13 METs were modified. 